\title{
Filtration Performance of Layering Masks and Face Coverings and the Reusability of Cotton Masks after Repeated Washing and Drying
}

\author{
Sumit Sankhyan ${ }^{1}$, Karen N. Heinselman², Peter N. Ciesielski ${ }^{2}$, Teresa Barnes ${ }^{2}$, \\ Michael E. Himmel ${ }^{2}$, Hannah Teed ${ }^{3}$, Sameer Patel ${ }^{1 \neq}$, Marina E. Vance ${ }^{1,3^{*}}$ \\ ${ }^{1}$ Department of Mechanical Engineering, University of Colorado Boulder, Boulder, CO 80309, \\ USA \\ ${ }^{2}$ Renewable Resources and Enabling Sciences Center, National Renewable Energy Laboratory, \\ Golden, CO 80401, USA \\ ${ }^{3}$ Environmental Engineering Program, University of Colorado Boulder, Boulder, CO 80309, USA
}

\section{ABSTRACT}

In this study, filtration efficiency of different respirators, face masks, and a 2-ply cotton handkerchief bandana was compared for particles in the size range of $60 \mathrm{~nm}-4 \mu \mathrm{m}$ under a "perfect fit" condition. The filtration efficiency at the most penetrating particle size of $0.3 \mu \mathrm{m}$ on average ranged from $83-99 \%$ for $\mathrm{N} 95$ and KN95 respirators, $42-88 \%$ for surgical masks, $16-23 \%$ for cloth masks, and $9 \%$ for bandana. We also investigated the effects of using double surgical masks or layering a cloth mask over various surgical masks in terms of their filtration characteristics. In most of these combinations, the filtration efficiency improved by $25 \%$ for particles $0.3-1 \mu \mathrm{m}$ in diameter without any substantial change in the filter quality factor when compared to the highest of the individual mask results. To investigate the reusability of cotton cloth masks, 2-layer cotton fabric sample coupons were machine washed and dried for 52 cycles leading to an increase in inhalation resistance $(\sim 20 \mathrm{~Pa})$ without affecting size-resolved filtration efficiency. Scanning electron microscopy revealed that washing and drying led to the gradual deconstruction of cotton fibers at the scale of several micrometers to hundreds of nanometers in the form of delamination of the fiber wall and fibrillation of the nanofiber constituents. Results indicate that cloth masks may be layered over surgical masks for additional benefits, and that cloth masks made out of cotton fabric can be washed and reused numerous times without a significant loss in filtration efficiency.

Keywords: COVID-19, Wildfire, Filter quality factor, PPE

‡ Present address: Department of Civil Engineering, IIT Gandhinagar, Gandhinagar, Gujarat 382355, India

\section{Publisher:}

Taiwan Association for Aerosol Research

ISSN: $1680-8584$ print

ISSN: 2071-1409 online

(c) Copyright: The Author(s).

This is an open access article distributed under the terms of the Creative Commons Attribution License (CC BY 4.0), which permits unrestricted use, distribution, and reproduction in any medium, provided the original author and source are cited.

\section{INTRODUCTION}

The coronavirus disease (COVID-19) worldwide pandemic, caused by the severe acute respiratory syndrome coronavirus 2 (SARS-CoV-2), led to a widespread healthcare supply and personal protective equipment (PPE) shortage, especially in terms of $\mathrm{N} 95$ respirators and surgical masks, for healthcare workers and the broad community (Ranney et al., 2020). This severe shortage led essential workers to reuse disposable PPE and for the public to turn to new suppliers and products, such as bandana and cloth masks, surgical masks, and KN95 respirators for everyday use.

Face coverings are now required or encouraged in many locations around the world (Rab et al., 2020; Yan et al., 2021). This stems from the important role that airborne transmission plays in the spread of COVID-19, as shown by empirical evidence in recent studies describing "super spreader events". A chorale rehearsal in which 53 of 61 members contracted COVID-19 demonstrated that the air was the most likely route of transmission (Miller et al., 2021). The investigation of an outbreak in a large German meat processing complex showed that transmission likely occurred 
via airborne route, over long distances $(\sim 8 \mathrm{~m})$ in a poorly ventilated space (Günther et al., 2020). Additionally, the ventilation system was shown to play a key role in an outbreak in a restaurant in China (Lu et al., 2020b).

There are several important aspects associated with the use of face coverings in the context of reducing respiratory disease transmission, namely: (1) inward filtration efficiency, providing protection to the wearer, (2) outward filtration efficiency, preventing the exhaled virus spread from the wearer, (3) inhalation resistance, or pressure drop across the material, and (4) how well the mask or face covering fits the wearer, preventing air shortcuts along the sides of the material.

Recent studies have demonstrated that the use of face masks reduces respiratory aerosol/droplet emissions and respiratory virus shedding (Asadi et al., 2020; Howard et al., 2021; Leung et al., 2020). Face masks, if worn consistently and correctly can also reduce the risk of respiratory virus transmission by acting as personal protection devices that filter out infectious respiratory droplets containing the virus (Sung et al., 2016; Zhang et al., 2013). However, the filtration performance of face masks depends on a variety of factors including number of filter material layers and their overall filtration efficiency, conditions in which they are being worn, and the fit or air seal that can be achieved on the wearer's face (Tcharkhtchi et al., 2021).

Face masks and respirators can also be useful for reducing $\mathrm{PM}_{2.5}$ exposure in regions affected by wildfires. Face masks with higher filtration efficiencies in the fine and ultrafine size ranges would be preferred in this scenario. Also, since the aerosol transmission of SARS-CoV-2 virus is likely to take place via respiratory droplets in the 1-4 $\mu \mathrm{m}$ size range, it is important to study the filtration performance of different face coverings over a large size range for their usage in the context of protection against fine PM exposure in addition to reducing COVID transmission (Chia et al., 2020; Pan et al., 2021; Santana et al., 2020).

Disposable surgical masks are more effective in reducing fine particulate matter $\left(\mathrm{PM}_{2.5}\right)$ exposure than cloth masks (Shakya et al., 2017). Previous studies demonstrated that face masks made out of layered fabrics are $35-45 \%$ efficient in removing submicron PM and in some cases, material combinations had higher filtration efficiencies than $\mathrm{N} 95$ respirators and surgical masks, albeit the substantially lower breathability of such combinations makes them an unsuitable option (O'Kelly et al., 2020). A similar study tested the penetrations levels of cloth masks and other filters against 20-1000 nm sized polydisperse Sodium Chloride ( $\mathrm{NaCl}$ ) aerosols and reported filtration efficiencies in the 40-90\% range (Rengasamy et al., 2010).

Although cloth masks may only provide marginal protection against submicron PM exposure, they are a reusable and potentially more sustainable approach than disposable masks and have been used widely by the public during the COVID-19 pandemic. The filtration efficiency of cloth masks can be optimized by layering different filter materials while maintaining similar breathability levels as commercially available masks. A study tested four cloth masks made using different combinations of Cotton, Lycra, and PTFE membrane layers and reported filtration efficiency for particles in the $0.1-1 \mu \mathrm{m}$ size range in the $23-88 \%$ range (Lu et al. , 2020a). Cloth masks made out of polyester, nylon, polypropylene or silk fabrics can even be triboelectrically charged to enhance their filtration efficiency (Zhao et al., 2020). Moreover, layering multiple masks, i.e., doublemasking, has become popular in the later months of the COVID-19 pandemic and is suggested to improve fit and filtration efficiency, although quantitative assessments of filtration efficiency and breathability lack in the current literature (Brooks, 2021).

The sustainability, reusability, and end-of-life of face coverings are important aspects of increased mask usage during the COVID pandemic. The increase in demand for disposable surgical masks (containing polymer nanofibers as filters) contributes to the already existing issue of microplastic pollution in terrestrial and aquatic environments (Aragaw, 2020). Cloth masks made out of cotton on the other hand can be reused by washing, are cheaper to make, can be produced locally in the absence of manufacturing facilities, and are biodegradable in the natural environment (Park et al., 2004; Salter, 2020). Regular washing of cloth masks also serves an important role in reducing the risk of infection due to increased moisture retention in case of their prolonged and repeated use (Asadi et al., 2020; Maclntyre et al., 2009).

In this work, we compare the filtration performance of different classes of respirators, face masks, and a 2-ply cotton handkerchief bandana in terms of PM-number based concentrations, PM-mass based filtration efficiency at the $300 \mathrm{~nm}$ particle diameter, and the resulting filter quality factor (QF). We also quantify the benefits of using double surgical masks or a combination 
of surgical mask and cloth mask compared to the filtration performance of a single mask. In order to investigate the potential for long-term reusability of cloth masks made out of cotton fabric, we characterize the effects of washing and drying these masks regularly on their filtration characteristics.

\section{METHODS}

\subsection{Materials Tested}

In this study we investigated N95 and KN95 respirators, surgical mask samples from 4 different manufacturers, 3 different types of commercially available cloth masks (one made entirely of cotton, and two that included a top layer made of polyester), a 2-ply cotton handkerchief bandana, as well as separate 2-layer cotton coupons prepared specifically to investigate wash/dry cycles (Fig. S1). We also investigated the use of double masks as a measure to achieve better filtration characteristics as compared to wearing a single mask. Surgical mask samples from three different manufacturers were mounted on the testing apparatus in duplicates. Additionally, identical cloth mask was also layered over these three different surgical mask brands to evaluate the improvement in filtration efficiency from this combination strategy. All samples were pre-conditioned in a chamber at $85 \pm 5 \%$ relative humidity and $38 \pm 2.5^{\circ} \mathrm{C}$ for $25 \pm 1$ hours before testing, according to the testing conditions and requirements of the NIOSH N95 filtration efficiency procedure (NIOSH, 2019a). After conditioning, samples were either tested immediately or sealed in an airtight container and tested within 10 hours.

\subsection{Wash/Dry Sample Coupons}

The wash/dry sample coupons were made of 2 layers of Kona cotton broadcloth, a 120-thread count, $100 \%$ cotton fabric commonly used for quilting. The reported fabric weight is $147.5 \mathrm{~g} \mathrm{~m}^{-2}$. The fabric underwent one hot wash and hot dry cycle before cutting, to pre-shrink the cotton. The fabric was then cut into $20 \times 20 \mathrm{~cm}$ squares, and 2 squares ( 1 light and 1 dark side) were placed right sides together and sewn around the edges with a $1 / 4$ " seam, leaving a $5 \mathrm{~cm}$ gap to flip the raw edge to the inside. Before flipping the coupon right side out, the corners were clipped to reduce bulk. The coupon was then flipped right side out, pressed into shape, and finally topstitched to seal up the hole left for flipping the raw edge in and reinforce the seams (Fig. S2).

\subsubsection{Wash/Dry Procedure}

Washing and drying was performed using a washer and dryer system (High-Efficiency Duet Steam, Whirlpool Corp., Benton Harbor, MI). For the wash cycle, the normal/casual setting was used, with the temperature adjusted to the hot wash/cold rinse option, to maximum temperature of around $\sim 50^{\circ} \mathrm{C}$. The laundry detergent used was a non-enzymatic (non-cellulase) detergent that contains biodegradable anionic and nonionic surfactants (Xtra Lasting ScentSations, Church \& Dwight Co., Inc.). For the dryer cycle, the heavy-duty setting was used, which automatically set the dry cycle temperature to Hot.

\subsection{Experimental Design}

Two experimental setups (Setup 1 and Setup 2) were used to investigate the filtration performance of the different masks and respirators over a wide range of particle sizes.

\subsubsection{Setup 1}

Setup 1 was used to characterize filtration efficiencies for particles in the size range of $60 \mathrm{~nm}-$ $450 \mathrm{~nm}$. Tests were performed in a $37.8 \mathrm{~m}^{3}$ test room (Fig. S3) and the testing conditions were adapted from the NIOSH N95 filtration efficiency procedure (NIOSH, 2019a). A fan was kept inside the room to ensure well-mixed conditions. The testing chamber was maintained at $\sim 22^{\circ} \mathrm{C}$ and $\sim 37 \%$ relative humidity. All instruments, the aerosol generation system and accessories, and pumps were kept outside the room. Copper (ID: $3 \mathrm{~mm}$ ) sample lines of equal length and diameter, but one with and one without a mask, ran in parallel to ensure that particle losses were equal in both the lines. A solution of $10 \%$ by weight of ammonium sulfate $\left[\left(\mathrm{NH}_{4}\right)_{2} \mathrm{SO}_{4}\right]$ in deionized water 
was prepared and placed in a Collison-type atomizer, operated at $\sim 32$ psi to generate an aerosol with a median diameter of $75 \pm 20 \mathrm{~nm}$. We used ammonium sulphate instead of sodium chloride, which is recommended by NIOSH (2019a), to avoid the risk of corrosion in stainless steel parts of the test setup. The aerosol stream passed through a diffusion dryer to remove water and an X-ray neutralizer to neutralize electrical surface charge before being injected into the testing chamber. Measurements in this setup were performed by a Scanning Mobility Particle Sizer (SMPS 3080, TSI Inc., MN, USA) outfitted with a water-based Condensation Particle Counter (CPC 3788, TSI Inc., MN, USA) and an Aerosol Particle Sizer Spectrometer (APS 3321, TSI Inc., MN, USA). The measurements from SMPS and APS were both exported at a resolution of 32 channels/decade for better agreement.

For each test run, which lasted 60 minutes, the atomizer operated for the first 15 minutes followed by 45 minutes of filtration efficiency test leading to $\sim 0.1 \mathrm{mg}$ particle loading on mask. During this period, particle concentrations in the test room and downstream of the sampling material were measured multiple times by switching between the blank line and the mask line with the help of a 3-way valve to generate a filtration efficiency over time with four replicates.

\subsubsection{Setup 2}

Setup 2 (Fig. S4) was used to characterize the filtration efficiencies of larger particles, in the $542 \mathrm{~nm}-4 \mu \mathrm{m}$ particle size range, using the same APS instrument as in Setup 1 . In this setup, a $1.7 \mathrm{~m}^{3}$ acrylic chamber containing a small fan to aid in aerosol mixing was used. This chamber was kept at $\sim 22^{\circ} \mathrm{C}$ and $\sim 30-40 \%$ relative humidity. A mixture of $5 \%$ by weight of $\left(\mathrm{NH}_{4}\right)_{2} \mathrm{SO}_{4}$ and $3-\mu \mathrm{m}$ and $7-\mu \mathrm{m}$ polystyrene latex spheres (PSL, Sigma-Aldrich, MO, USA) in deionized water was used to generate particles using a medical-grade nebulizer (Sigma-Aldrich, MO, USA). The aerosol stream passed through a diffusion dryer to remove water before entering the testing chamber. Since previous studies have reported similar filtration efficiencies with and without using a neutralizer, we did not use an X-ray neutralizer in this setup to avoid sampling losses for larger particles (Drewnick et al., 2021; Pan et al., 2021). Example particle concentrations inside the chambers during the testing duration for both Setup 1 and Setup 2 are shown in Figs. S5 and S6, respectively.

For both testing setups, respirators were mounted on a mannequin head, a Leland Legacy pump (SKC Ltd., DT, UK) was used to maintain a sampling rate of $15 \mathrm{~L} \mathrm{~min}^{-1}$ in both mask and blank line. This flow rate is comparable to human breathing rate at a light intensity activity level for most of the age groups (U.S. EPA, 2011). Assuming the surface area of a standard N95 respirator to be $175 \mathrm{~cm}^{2}$ (Roberge et al., 2010), the corresponding face velocity through the respirator was calculated to be $1.4 \mathrm{~cm} \mathrm{~s}^{-1}$. The face velocity used in our study lies in the range of $0.5-25 \mathrm{~cm} \mathrm{~s}^{-1}$ based on the filtration efficiency test standards used by the American Society for Testing and Materials and the U.S. Food and Drug Administration (ASTM International, 2017; FDA, 2018; O'Kelly et al., 2020). All the face masks, bandana, and washing study coupon samples were mounted on a 4-inch $(\sim 10 \mathrm{~cm})$ diameter stainless steel funnel instead of mannequin head to achieve an air tight seal. For funnel-mounted samples, a sampling flow rate of $6.7 \mathrm{~L} \mathrm{~min}^{-1}$ was maintained to achieve the same flow velocity of $1.4 \mathrm{~cm} \mathrm{~s}^{-1}$ through the filter cross-section.

For both testing setups, the filtration efficiency corresponding to a given particle size $D_{p}$ was calculated using Eq. (1):

$F E\left(D_{p}\right)=\frac{C_{\text {blank }}-C_{\text {mask }}}{C_{\text {blank }}}$

where $C_{\text {blank }}$ is the average concentration of the blank line measured before and after the mask line concentration $\left(C_{\text {mask }}\right.$ ). This method of estimation accounts for temporal variations in chamber concentrations and has been used by several studies in the past (Konda et al., 2020; Morais et al., 2021; Pan et al., 2021).

For double mask combinations, we compared the experimental results with the results from a simple model based on the classical filtration theory for filters to check whether these mask combinations can be treated as series of filters connected in series. The filtration efficiency of a given mask combination was calculated using the Eq. (2) (Hinds, 1999): 


$$
T_{c}=T_{m_{1}} \times T_{m_{2}}
$$

where $T_{c}$ is the particle transmission through a given double mask combination, $T_{m_{1}}$ and $T_{m_{2}}$ are particle transmission through mask 1 and mask 2 respectively.

Particle transmission $T$ is further related to the filtration efficiency (FE) by the following equation:

$T=1-\frac{F E(\%)}{100}$

\subsection{Inhalation Resistance}

The test for the determination of inhalation resistance was performed according to NIOSH procedure TEB-APR-STP-0007-508 (NIOSH, 2019b), which lists a $35 \mathrm{~mm}$ water-column height limit for $\mathrm{N} 95$ respirators. Each sample was affixed to a funnel $(4.5 \mathrm{~cm}$ diameter) in the case of masks and flat filter samples, or taped to a mannequin head in the case respirators. A flow rate corresponding to an $8 \mathrm{~cm} \mathrm{~s}^{-1}$ face velocity (corresponding to 85 LPM flow rate through an N95 respirator) was pulled through the material and the pressure drop was measured using a digital manometer (Datum 2239-1, Setra Systems Inc., Boxborough, MA, USA).

\subsubsection{Filter quality factor (QF)}

The filter quality factor (QF) which relates the pressure drop of the material with the PM massbased filtration efficiency was calculated using Eq. (4) (Hinds, 1999; Podgórski et al., 2006):

$Q F\left(D_{p}\right)=-\frac{\left(\ln \left(1-F E\left(D_{p}\right)\right)\right)}{\Delta P}$

where $Q F\left(D_{p}\right)$ is the filtration efficiency at particle size $D_{p}$ and $\Delta P$ is the pressure drop across the material. The value of $Q F(0.3 \mu \mathrm{m})$ has been used in previous studies to compare filtration performance among different filters so we will use the same values in our study for comparison. High values of $Q F$ for a given filter imply that the filter can give better filtration performance with greater breathability.

\subsection{Scanning Electron Microscopy Analyses}

In order to characterize morphological changes on the cotton fabric due to repeated washing and drying, we visualized those samples through scanning electron microscopy (SEM) using a FEI Quanta 400 FEG instrument (FEI, Hillsboro, OR). Single layer fabric samples of $\sim 0.5 \mathrm{~cm}^{2}$ were cut from larger fabric samples after 0,24 , and 52 wash/dry cycles. The specimens were mounted on aluminum stubs with double-sided conductive carbon tape and sputter-coated with $12 \mathrm{~nm}$ of iridium. Imaging was performed with beam accelerating voltage of $5 \mathrm{keV}$ at magnifications below $1000 \times$, accelerating voltage of $10 \mathrm{keV}$ was used at higher magnifications.

\section{RESULTS AND DISCUSSION}

\subsection{Size-resolved Filtration Efficiencies}

The results for the size-resolved filtration efficiency of different samples are shown in Fig. 1. N95 and KN95 respirators presented highest filtration efficiencies (> 95\%) throughout the size range investigated $(60 \mathrm{~nm}-4 \mu \mathrm{m})$, as expected. The filtration efficiency curve for rest of the masks and bandana samples exhibited the traditional " $u$ " shape with minima in the accumulation mode size range $(0.1-1 \mu \mathrm{m})$, except for surgical mask $A$, which presented high filtration efficiencies $(>85 \%)$ through the entire size range. For most double mask combinations, the average filtration efficiency increased by $\sim 25 \%$ for particles in the accumulation mode size range for a given mask combination when compared to the highest of the individual mask results. The results for overall filtration efficiency in terms of total PM 2.5 number and mass, and in terms of PM mass at $300 \mathrm{~nm}$ for all the samples collected using Setup 1 are presented in supplemental Table S1. 

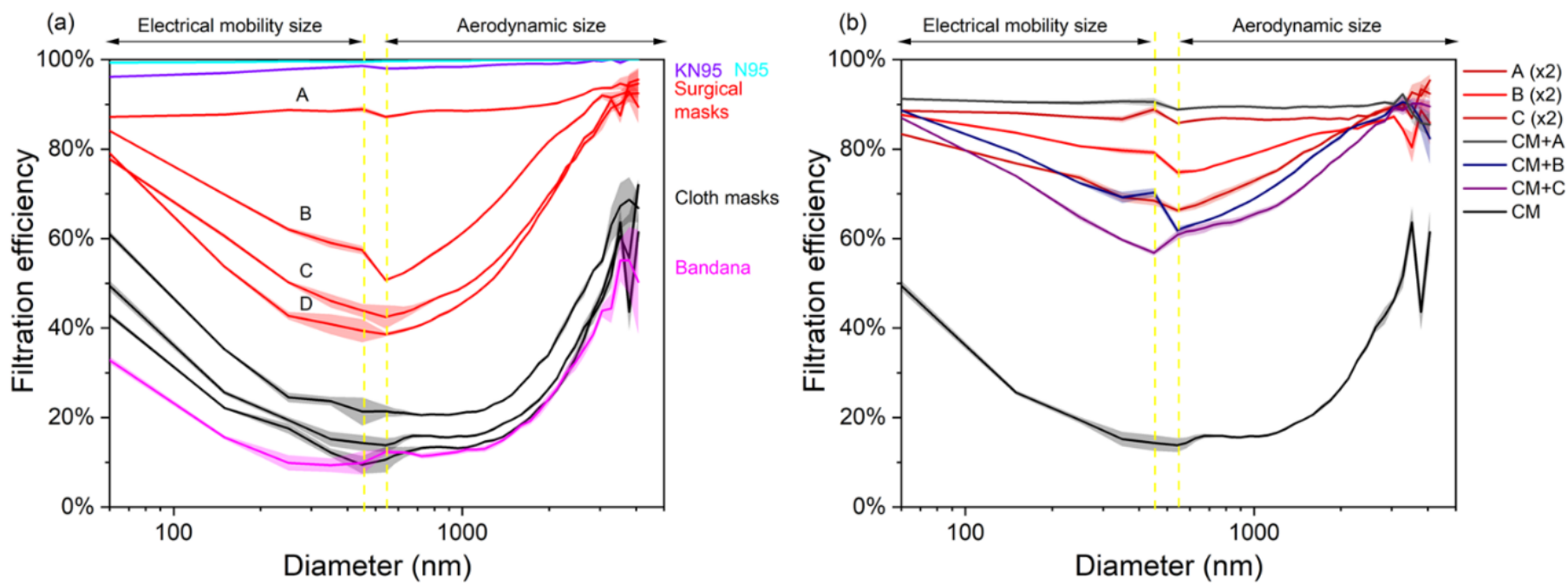

Fig. 1. Panel (a) Size-resolved filtration efficiency measured using particle number-based concentrations for different respirators, masks, and bandana. Panel (b) Size-resolved filtration efficiency for different mask combinations, where A, B, and C are the same surgical masks as shown in panel (a) and CM is a cloth mask used for layering. The shaded region represents standard errors ( $\mathrm{n}$ $=4)$.

While the filtration efficiency curve for the N95 respirators remained constant at $\sim 99 \%$ for the entire size range, the KN95 respirators presented slightly lower filtration efficiencies (from 96\% to $98 \%$ ) for particles $60-400 \mathrm{~nm}$, and remained constant at $98 \%$ for larger particles. Filtration efficiency results for $\mathrm{N} 95$ and KN95 respirators were used to verify the testing setup adapted for this study, and they also agree well with results from similar studies based on NIOSH respirator testing (Lu et al., 2020a; Morais et al., 2021; Rengasamy et al., 2010; Zangmeister et al., 2020).

The cloth masks and 2-layer bandana presented similar average filtration efficiencies, between 30-60\% for particles smaller than $100 \mathrm{~nm}$ size, 10-25\% for particles sized between $300 \mathrm{~nm}$ and $1 \mu \mathrm{m}$, and $12-68 \%$ for particles sized 1-4 $\mu \mathrm{m}$. The cloth mask that was made of all-cotton layers had a slightly lower filtration efficiency compared to the other two cloth masks, which both included a polyester top layer. For surgical masks, the average filtration efficiency for mask $A$ ranged between 87-95\% showing a slight overall linear increase for particle sizes in the range of $60 \mathrm{~nm}-4 \mu \mathrm{m}$, whereas the filtration efficiency of samples B-D was $70-85 \%$ for particles smaller than $100 \mathrm{~nm}$ size, dropped to $40-60 \%$ for particles between $300 \mathrm{~nm}-1 \mu \mathrm{m}$, and increased to $45-$ $94 \%$ particles in the size range of $1-4 \mu \mathrm{m}$. These results agree well with previous studies reporting filtration efficiencies in the 50-80\% range for submicron particles for surgical masks, although these studies employed higher test flow rates, commonly used for NIOSH test method of N95 certification (Pan et al., 2021; Zangmeister et al., 2020).

The overall higher filtration efficiency measured for surgical mask $A$ could be attributed to the presence of a "submicron filtration layer for dust protection" middle layer (as labeled on the packaging box), whereas no such layer was mentioned on the packaging of the other three samples. Since all 4 mask samples had no discernible features based on visual inspection, surgical mask A could have an electrically-charged filter in the middle which would lead to higher filtration efficiency in the accumulation mode size range when compared to non-charged filter materials (Wang, 2001).

For double-mask combinations (Fig. 1(b)), our results showed that doubling surgical masks led to an overall improvement in the filtration efficiencies of masks $B$ and $C$ whereas no such improvement was seen in the case of mask $A$, which could imply that mask $A$ is at the upper limit of filtration performance which couldn't be improved further by doubling. Layering a cloth mask (CM) over each of these surgical masks led to higher filtration efficiency curves particularly in the accumulation size range when compared to the individual cloth and surgical masks. These results suggest that the combination of a cloth mask over a surgical mask is likely to be a better alternative than wearing just a single mask.

These experimental results agreed with theoretical calculations for particle transmission 
through most double-mask combinations based on classical filtration theory. Filtration efficiency curves showing good overall agreement in combinations can be observed in Fig. S7. A better overall agreement was observed for cloth + surgical mask combinations (RMSE = 4-6\%) compared to the double surgical mask combinations (RMSE $=5-11 \%$ ). The higher values of RMSE in case of double surgical mask combinations could be attributed to the inference between individual filter layers resulting in lower filtration efficiencies than predicted (Drewnick et al., 2021).

It is important to bring these results under perspective because these tests are valid only for a "perfect fit" condition, i.e., assuming that there are no gaps between the mask and the face of the wearer, so it would be essential that the surgical mask is properly sealed and that the additional cloth mask or surgical mask does not affect that fit in any way. Previous studies have reported that a drop of $>50 \%$ in filtration efficiency can occur in the presence of minor leaks when compared with a perfect seal condition (Drewnick et al., 2021; Konda et al., 2020).

\subsection{Filtration Efficiency, Inhalation Resistance, and Resulting Filter Quality Factors}

Fig. 2 shows the PM-mass based filtration efficiency for particles $300 \mathrm{~nm}$ in diameter as a function of their inhalation resistance or pressure drop, and the resulting filter QF value.

The inhalation resistance of $\mathrm{N} 95$ and KN95 respirators was in the 125-200 Pa range, below the NIOSH limit of $343 \mathrm{~Pa}$ for N95 respirators (NIOSH, 2019b) and the corresponding average PM based filtration efficiency at $300 \mathrm{~nm}$ size was in the 83-99\% range. The inhalation resistance for surgical masks was much lower, in the 35-55 Pa range and the corresponding PM-mass based filtration efficiency at $300 \mathrm{~nm}$ size was calculated to be $88 \%$ on average for surgical mask $A$ and $42-62 \%$ for the remaining surgical mask samples. As discussed in the previous section, the presence of a "submicron dust filtration layer" in surgical mask A may have led to a higher filtration efficiency without increasing the pressure drop considerably when compared to other surgical masks investigated. The lowest results for filtration efficiency at $300 \mathrm{~nm}(<25 \%)$ were measured for cloth masks and the bandana and the average pressure drop was measured in the range of 10$50 \mathrm{~Pa}$. In general, a trend can be observed in Fig. 2(a), with lower pressure drop values associated with lower filtration efficiencies.

In the case of double surgical masks and the combination of cloth + surgical masks, the inhalation resistance increases were also accompanied by improvements in the filtration behavior relative to

(a)

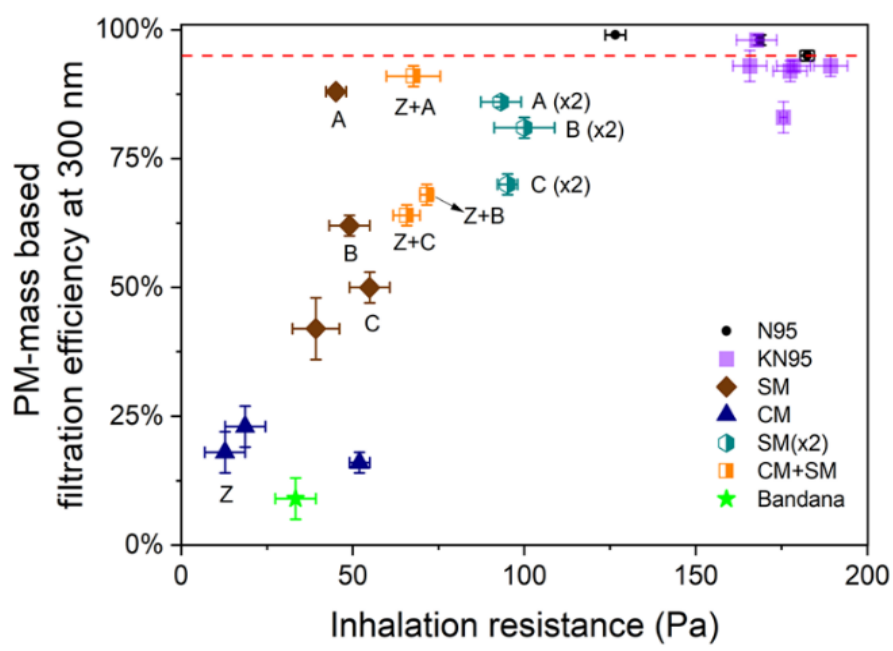

(b)

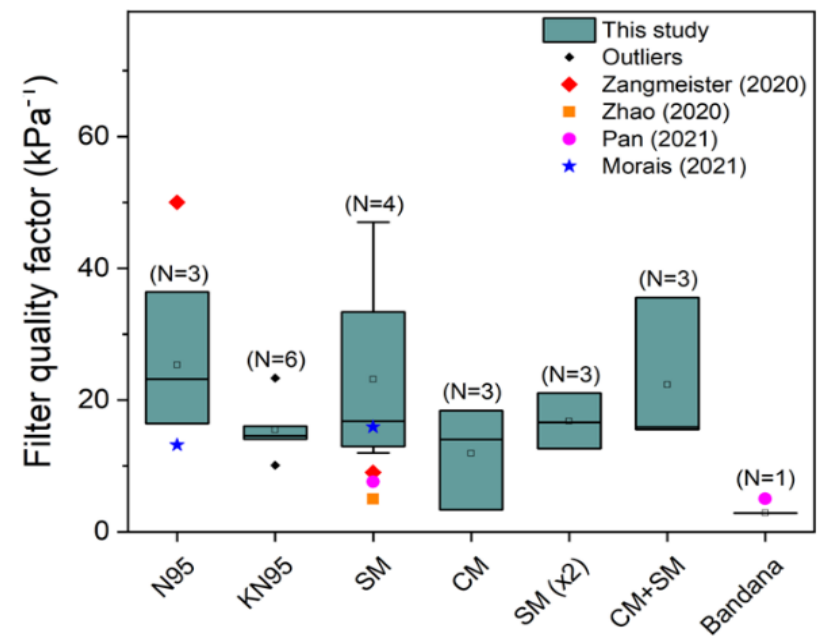

Fig. 2. PM-mass based filtration efficiency at $300 \mathrm{~nm}$ as a function of the inhalation resistance of different respirators, masks, and bandana is shown in panel (a). The whiskers represent standard deviations ( $n=4$ for filtration efficiency tests and $n=3$ for pressure drop tests). The cloth mask and the surgical mask samples along with their different combinations have been labeled as Z, A, B, and C respectively. Panel (b) shows the filter quality factors calculated for different sample categories. The squares represent the mean values whereas the median is represented by a horizontal line. The corresponding filter quality factor results from previous studies are also included in panel (b) for comparison. 
individual cloth mask and surgical mask results. There is one exception, in the case of surgical mask A, where doubling didn't improve the filtration efficiency at $300 \mathrm{~nm}$ ( $88 \%$ for the single mask versus $86 \pm 1 \%$ for the double mask) but increased the inhalation resistance, from $45 \pm 3 \mathrm{~Pa}$ for a single mask to $93 \pm 6 \mathrm{~Pa}$ for a double mask. As expected, doubling up surgical masks roughly doubled the inhalation resistance. Layered masks, either cloth + surgical or double surgical mask combinations, demonstrated lower inhalation resistance values and lower filtration efficiencies than those for $\mathrm{N} 95$ or KN95 respirators.

In terms of the mean QF, N95 respirators $\left(25 \mathrm{kPa}^{-1}\right)$ performed better than KN95 respirators $\left(15 \mathrm{kPa}^{-1}\right)$. Among face masks and coverings, surgical masks had the highest mean QF value $\left(23 \mathrm{kPa}^{-1}\right)$ followed by cloth masks $\left(12 \mathrm{kPa}^{-1}\right)$ and the bandana $\left(3 \mathrm{kPa}^{-1}\right)$. The surgical masks investigated in this study exhibited higher QF than KN95 respirators due to their relatively low inhalation resistance ( $\sim 88 \mathrm{~Pa}$ compared to $\sim 180$ Pa for KN95 respirators).

In the case of mask combinations, the QF value for doubling surgical mask $\mathrm{A}\left(21 \mathrm{kPa}^{-1}\right)$ and layering it with a cloth mask $\left(36 \mathrm{kPa}^{-1}\right)$ decreased when compared to wearing surgical mask $A$ on its own $\left(47 \mathrm{kPa}^{-1}\right)$ but these QF values were still higher than that of the cloth mask $\left(18 \mathrm{kPa}^{-1}\right)$. A much smaller decrease $\left(\sim 3 \mathrm{kPa}^{-1}\right)$ was observed for both doubling surgical mask $B$ and for layering it with a cloth mask, compared to the corresponding QF value for the single surgical mask $B$ $\left(20 \mathrm{kPa}^{-1}\right)$. On the other hand, the $\mathrm{QF}$ values for a single and double surgical mask $\mathrm{C}$ were similar $\left(\sim 12 \mathrm{kPa}^{-1}\right)$ and the QF value increased by $4 \mathrm{kPa}^{-1}$ while layering this mask with a cloth mask. These results indicate that doubling or layering can be a better option than single masks, especially when surgical masks with lower filtration efficiencies are available.

\subsection{Effects of Washing and Drying on Cloth Masks}

The effect of repeated washing and drying on the filtration behavior and inhalation resistance of cotton fabric is shown in Fig. 3. An overall increase in inhalation resistance was observed as the fabric sample underwent 52 hot wash/dry cycles, but overall filtration efficiency did not change significantly with wash/dry cycles.

Except for $a \sim 10 \%$ decrease $(p<0.01$ ) in filtration efficiency at $2.5 \mu \mathrm{m}$ from 1 to 7 wash/dry cycle, the PM-mass based filtration efficiency results didn't show any major fluctuations as the number of washing and drying cycles were incrementally increased to 52 cycles (Fig. 3(b)). A previous study on the reusability of self-developed cloth masks made up of cotton, Lycra, and polypropylene fabric also reported insignificant changes $(p>0.05)$ in average filtration efficiency

(a)

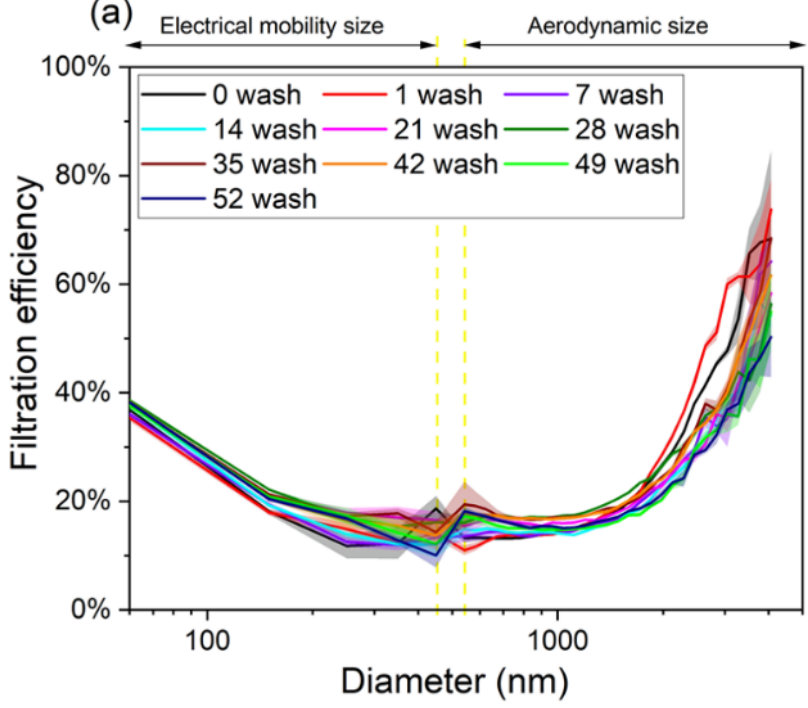

(b)

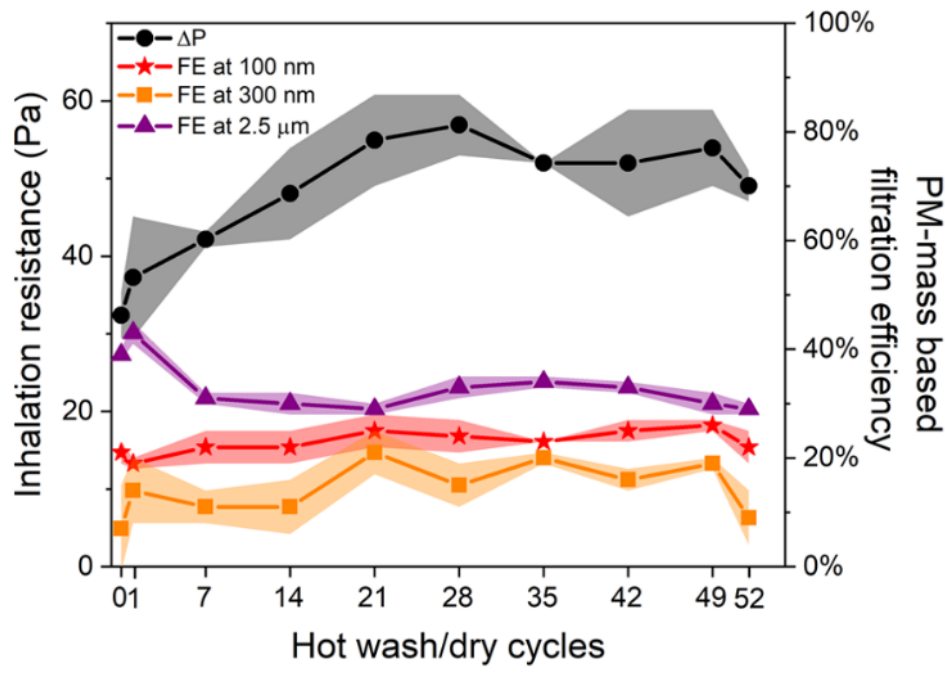

Fig. 3. Size-resolved filtration efficiency calculated as a function of particle number distribution is shown in panel (a). The shaded region represents standard error $(n=4)$. Panel $(b)$ shows the inhalation resistance (black, left-hand y-axis) and PM-mass based filtration efficiency at 3 different particle sizes (plotted on the right-hand y-axis) of fabric material as a function of number of hot wash/dry cycles. The shaded region represents standard deviation values. 
between an unwashed sample one that was washed and air-dried 30 times for accumulation mode particles (Lu et al., 2020a).

In terms of the effect of wash/dry cycles on breathability, the inhalation resistance increased on average by $\sim 17 \mathrm{~Pa}$ between zero and 52 wash/dry cycles $(p=0.002)$. The inhalation resistance for the unwashed sample coupon was measured to be $32 \pm 2 \mathrm{~Pa}$ and increased to a maximum inhalation resistance of $57 \pm 5 \mathrm{~Pa}$ for the 28 -hot wash/dry cycle sample coupon. Afterwards, the curve somewhat plateaus at $53 \pm 5 \mathrm{~Pa}$.

The resulting QF calculated for the cotton fabric samples as a function of wash/dry cycles remained below $5 \mathrm{kPa}^{-1}$ and didn't change significantly between the unwashed and the sample that underwent 52 wash/dry cycles (Fig. S8). This is likely because the QF value is more sensitive to changes in $\mathrm{FE}$ than in $\Delta \mathrm{P}$ and, although a trend was observed for $\Delta \mathrm{P}$ throughout the repeated wash/dry cycles, the same did not occur for the FE at $300 \mathrm{~nm}$. Based on these results, we conclude that repeated washing and drying of cloth masks made of cotton fabric did not alter their filtration characteristics in a major way, thus indicating a favorable potential for the reusability of these products.

\subsubsection{SEM analyses of wash/dry sample coupons}

Scanning electron microscopy (SEM) was used to investigate changes in micro- and nanoscale morphology of the cotton fabric. Representative micrographs of cotton fabric subjected to 0,28 , and 52 washing cycles are presented in Fig. 4.

The as-received cotton fabric exhibited largely intact bundles of cotton fibers (Fig. 4(a)) with relatively smooth surface texture (Fig. $4\left(a^{\prime}\right)$ ). After 28 wash/dry cycles, the fiber bundles display a larger population of partially liberated fibers. Higher magnification images reveal deconstruction of individual fibers at the scale of hundreds of nanometers to several micrometers in the form of delamination of the fiber wall and fibrillation of the nanofiber constituents (Fig. $4\left(b^{\prime}\right)$ ). This pattern of deconstruction results from the hierarchical ordering of cellulose composing cotton cell walls and is similar to the morphology observed when fibers from other herbaceous species such as maize and switchgrass are subjected to thermochemical treatments (Ciesielski et al., 2014; Karp et al., 2015). Fabric samples that had experienced 52 wash/dry cycles showed an even greater population of partially liberated fibers (Fig. $4(\mathrm{c})$ ) and more extreme degrees of nanoscale delamination and fibrillation (Fig. 4(c')). These modes of deconstruction increase porosity of the macrofibers and liberate nanoscale cellulose bundles which may be subjected to bending stress in a convective flow field and likely contribute to the increased pressure drop observed as washdry cycles progress (Ciesielski et al., 2019; Hinkle et al., 2015).
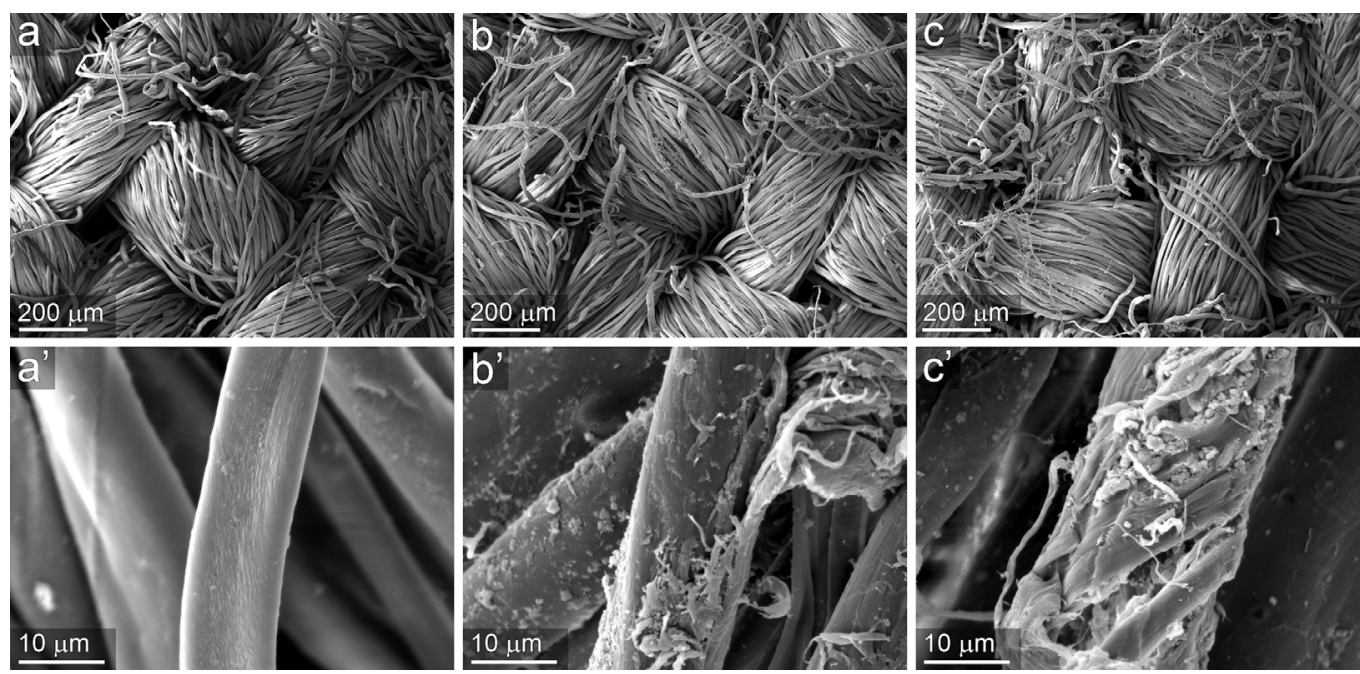

Fig. 4. Scanning electron micrographs of cotton fabric subjected to varying numbers of wash/dry cycles: as received $\left(a, a^{\prime}\right), 28$ cycles $\left(b, b^{\prime}\right)$, and 52 cycles $\left(c, c^{\prime}\right)$. Some cotton fibers displayed evidence of micro- and nanoscale deconstruction after 28 wash cycles. Both the population of deconstructed fibers and the extent of deconstruction increased further after 52 cycles. 
Recent medical and environmental events across the globe have dramatically increased the demand for use of face coverings as personal filtration devices. This work brings forth results of interest to the science community as well as the general public, such as the reusability of cloth masks after repeated washing and drying and the effects of overlaying multiple masks on their performance. In this study, size-resolved filtration efficiencies in the size range of $60 \mathrm{~nm}-4 \mu \mathrm{m}$ were investigated under perfect seal condition for different face covering options available in the market. The filtration efficiency at the most penetrating particle size of $0.3 \mu \mathrm{m}$ on average ranged from $83-99 \%$ for $\mathrm{N} 95$ and KN95 respirators, 42-88\% for surgical masks, 16-23\% for cloth masks, and $9 \%$ for bandana. The study also presents a positive argument for using a surgical mask with an electrically charged middle layer due to its superior filtration performance as compared to other samples, especially in the accumulation mode particle size range. This work has also shown that doubling surgical masks or layering a cloth mask over surgical mask can be a better option than single masks, especially when only surgical masks with lower filtration efficiencies are available. Results from the washing study show that filtration characteristics of cloth fabric did not change significantly despite the delamination of the fiber wall and fibrillation of the nanofiber constituents observed during SEM analysis. Since cotton masks offer a biodegradable and washable alternative to commonly used disposable surgical masks, the results of this work points towards an a more sustainable path to mask wearing.

\section{ACKNOWLEDGMENTS}

Research was supported by the DOE Office of Science through the National Virtual Biotechnology Laboratory, a consortium of DOE national laboratories focused on response to COVID-19, with funding provided by the Coronavirus CARES Act. The authors also thank numerous service clients who agreed to share their samples and corresponding results anonymously.

\section{SUPPLEMENTARY MATERIAL}

Supplementary material for this article can be found in the online version at https://doi. org/10.4209/aaqr.210117

\section{REFERENCES}

Aragaw, T.A. (2020). Surgical face masks as a potential source for microplastic pollution in the COVID-19 scenario. Mar. Pollut. Bull. 159, 111517. https://doi.org/10.1016/j.marpolbul.2020. 111517

Asadi, S., Cappa, C.D., Barreda, S., Wexler, A.S., Bouvier, N.M., Ristenpart, W.D. (2020). Efficacy of masks and face coverings in controlling outward aerosol particle emission from expiratory activities. Sci. Rep. 10, 15665. https://doi.org/10.1038/s41598-020-72798-7

ASTM International (2017). Standard Test Method for Determining the Initial Efficiency of Materials Used in Medical Face Masks to Penetration by Particulates Using Latex Spheres. ASTM F2299/F2299M-03; ASTM International, West Conshohocken, PA, https://doi.org/10.15 20/F2299_F2299M-03R17

Brooks, J.T. (2021). Maximizing Fit for Cloth and Medical Procedure Masks to Improve Performance and Reduce SARS-CoV-2 Transmission and Exposure, 2021 (No. 70). Centers for Disease Control and Prevention.

Chia, P.Y., Coleman, K.K., Tan, Y.K., Ong, S.W.X., Gum, M., Lau, S.K., Lim, X.F., Lim, A.S., Sutjipto, S., Lee, P.H., Son, T.T., Young, B.E., Milton, D.K., Gray, G.C., Schuster, S., Barkham, T., De, P.P., Vasoo, S., Chan, M., Ang, B.S.P., et al. (2020). Detection of air and surface contamination by SARS-CoV-2 in hospital rooms of infected patients. Nat. Commun. 11, 2800. https://doi.org/10. 1038/s41467-020-16670-2

Ciesielski, P.N., Wagner, R., Bharadwaj, V.S., Killgore, J., Mittal, A., Beckham, G.T., Decker, S.R., 
Himmel, M.E., Crowley, M.F. (2019). Nanomechanics of cellulose deformation reveal molecular defects that facilitate natural deconstruction. Proc. Natl. Acad. Sci. U.S.A. 116, 9825-9830. https://doi.org/10.1073/pnas.1900161116

Ciesielski, P.N., Wang, W., Chen, X., Vinzant, T.B., Tucker, M.P., Decker, S.R., Himmel, M.E., Johnson, D.K., Donohoe, B.S. (2014). Effect of mechanical disruption on the effectiveness of three reactors used for dilute acid pretreatment of corn stover Part 2: Morphological and structural substrate analysis. Biotechnol. Biofuels 7, 47. https://doi.org/10.1186/1754-6834-7-47

Drewnick, F., Pikmann, J., Fachinger, F., Moormann, L., Sprang, F., Borrmann, S. (2021). Aerosol filtration efficiency of household materials for homemade face masks: Influence of material properties, particle size, particle electrical charge, face velocity, and leaks. Aerosol Sci. Technol. 55, 63-79. https://doi.org/10.1080/02786826.2020.1817846

Günther, T., Czech-Sioli, M., Indenbirken, D., Robitaille, A., Tenhaken, P., Exner, M., Ottinger, M., Fischer, N., Grundhoff, A., Brinkmann, M.M. (2020). SARS-CoV-2 outbreak investigation in a German meat processing plant. EMBO Mol. Med. 12, e13296. https://doi.org/10.15252/emm m.202013296

Hinds, W.C. (1999). Aerosol technology: properties, behavior, and measurement of airborne particles. Wiley.

Hinkle, J.D., Ciesielski, P.N., Gruchalla, K., Munch, K.R., Donohoe, B.S. (2015). Biomass accessibility analysis using electron tomography. Biotechnol. Biofuels 8, 212. https://doi.org/10.1186/s130 68-015-0395-8

Howard, J., Huang, A., Li, Z., Tufekci, Z., Zdimal, V., Westhuizen, H.-M. van der, Delft, A. von, Price, A., Fridman, L., Tang, L.H., Tang, V., Watson, G.L., Bax, C.E., Shaikh, R., Questier, F., Hernandez, D., Chu, L.F., Ramirez, C.M., Rimoin, A.W. (2021). An evidence review of face masks against COVID-19. Proc. Natl. Acad. Sci. U.S.A. 118, e2014564118. https://doi.org/10.1073/pnas.2014 564118

Karp, E.M., Resch, M.G., Donohoe, B.S., Ciesielski, P.N., O’Brien, M.H., Nill, J.E., Mittal, A., Biddy, M.J., Beckham, G.T. (2015). Alkaline Pretreatment of Switchgrass. ACS Sustain. Chem. Eng. 3, 1479-1491. https://doi.org/10.1021/acssuschemeng.5b00201

Konda, A., Prakash, A., Moss, G.A., Schmoldt, M., Grant, G.D., Guha, S. (2020). Aerosol filtration efficiency of common fabrics used in respiratory cloth masks. ACS Nano. 14, 6339-6347. https://doi.org/10.1021/acsnano.0c03252

Leung, N.H.L., Chu, D.K.W., Shiu, E.Y.C., Chan, K.-H., McDevitt, J.J., Hau, B.J.P., Yen, H.L., Li, Y., Ip, D.K.M., Peiris, J.S.M., Seto, W.H., Leung, G.M., Milton, D.K., Cowling, B.J. (2020). Respiratory virus shedding in exhaled breath and efficacy of face masks. Nat. Med. 26, 676-680. https://doi.org/10.1038/s41591-020-0843-2

Lu, H., Yao, D., Yip, J., Kan, C.W., Guo, H. (2020a). Addressing COVID-19 spread: Development of reliable testing system for mask reuse. Aerosol Air Qual. Res. 20, 2309-2317. https://doi.org/ 10.4209/aaqr.2020.06.0275

Lu, J., Gu, J., Li, K., Xu, C., Su, W., Lai, Z., Zhou, D., Yu, C., Xu, B., Yang, Z. (2020b). COVID-19 Outbreak Associated with Air Conditioning in Restaurant, Guangzhou, China, 2020. Emerg. Infect. Dis. 26, 1628-1631. https://doi.org/10.3201/eid2607.200764

MacIntyre, C.R., Cauchemez, S., Dwyer, D.E., Seale, H., Cheung, P., Browne, G., Fasher, M., Wood, J., Gao, Z., Booy, R., Ferguson, N. (2009). Face mask use and control of respiratory virus transmission in households. Emerg. Infect. Dis. 15, 233-241. https://doi.org/10.3201/eid1502. 081167

Miller, S.L., Nazaroff, W.W., Jimenez, J.L., Boerstra, A., Buonanno, G., Dancer, S.J., Kurnitski, J., Marr, L.C., Morawska, L., Noakes, C. (2021). Transmission of SARS-CoV-2 by inhalation of respiratory aerosol in the Skagit Valley Chorale superspreading event. Indoor Air 31, 314-323. https://doi.org/10.1111/ina.12751

Morais, F.G., Sakano, V.K., Lima, L.N. de, Franco, M.A., Reis, D.C., Zanchetta, L.M., Jorge, F., Landulfo, E., Catalani, L.H., Barbosa, H.M.J., John, V.M., Artaxo, P. (2021). Filtration efficiency of a large set of COVID-19 face masks commonly used in Brazil. Aerosol Sci. Technol. 55, 10281041. https://doi.org/10.1080/02786826.2021.1915466

National Institute for Occupational Safety \& Health (NIOSH) (2019a). Determination of particulate filter efficiency level for N95 series filters against solid particulates for non-powered, air-purifying respirators standard testing procedure (STP). (No. TEB-APR-STP-0059). Pittsburgh, MA, USA. 
National Institute for Occupational Safety \& Health (NIOSH) (2019b). Determination of inhalation resistance test, air-purifying respirators. Standard Testing Procedure (STP). (No. TEB-APR-STP0007). Pittsburgh, MA, USA.

O'Kelly, E., Pirog, S., Ward, J., Clarkson, P.J. (2020). Ability of fabric face mask materials to filter ultrafine particles at coughing velocity. BMJ Open 10, e039424. https://doi.org/10.1136/bmjo pen-2020-039424

Pan, J., Harb, C., Leng, W., Marr, L.C. (2021). Inward and outward effectiveness of cloth masks, a surgical mask, and a face shield. Aerosol Sci. Technol. 55, 718-733. https://doi.org/10.1080/02 786826.2021.1890687

Park, C.H., Kang, Y.K., Im, S.S. (2004). Biodegradability of cellulose fabrics. J. Appl. Polym. Sci. 94, 248-253. https://doi.org/10.1002/app.20879

Podgórski, A., Bałazy, A., Gradoń, L. (2006). Application of nanofibers to improve the filtration efficiency of the most penetrating aerosol particles in fibrous filters. Chem. Eng. Sci. 61, 68046815. https://doi.org/10.1016/j.ces.2006.07.022

Rab, S., Javaid, M., Haleem, A., Vaishya, R. (2020). Face masks are new normal after COVID-19 pandemic. Diabetes Metab. Syndr. Clin. Res. Rev. 14, 1617-1619. https://doi.org/10.1016/j.d sx.2020.08.021

Ranney, M.L., Griffeth, V., Jha, A.K. (2020). Critical supply shortages-The need for ventilators and personal protective equipment during the Covid-19 pandemic. N. Engl. J. Med. 382, e41. https://doi.org/10.1056/NEJMp2006141

Rengasamy, S., Eimer, B., Shaffer, R.E. (2010). Simple respiratory protection--evaluation of the filtration performance of cloth masks and common fabric materials against $20-1000 \mathrm{~nm}$ size particles. Ann. Occup. Hyg. 54, 789-798. https://doi.org/10.1093/annhyg/meq044

Roberge, R.J., Bayer, E., Powell, J.B., Coca, A., Roberge, M.R., Benson, S.M. (2010). Effect of exhaled moisture on breathing resistance of N95 filtering facepiece respirators. Ann. Occup. Hyg. 54, 671-677. https://doi.org/10.1093/annhyg/meq042

Salter, S. (2020). Reinventing cloth masks in the face of pandemics. Risk Anal. 41, 731-744. https://doi.org/10.1111/risa.13602

Santana, F., Fischer, S., Jaeger, M., Wong-Parodi, G. (2020). Responding to simultaneous crises: Communications and social norms of mask behavior during wildfires and COVID-19. Environ. Res. Lett. 15, 111002. https://doi.org/10.1088/1748-9326/abba55

Shakya, K.M., Noyes, A., Kallin, R., Peltier, R.E. (2017). Evaluating the efficacy of cloth facemasks in reducing particulate matter exposure. J. Expo. Sci. Environ. Epidemiol. 27, 352-357. https://doi.org/10.1038/jes.2016.42

Sung, A.D., Sung, J.A.M., Thomas, S., Hyslop, T., Gasparetto, C., Long, G., Rizzieri, D., Sullivan, K.M., Corbet, K., Broadwater, G., Chao, N.J., Horwitz, M.E. (2016). Universal Mask usage for reduction of respiratory viral infections after stem cell transplant: A prospective trial. Clin. Infect. Dis. Off. Publ. Infect. Dis. Soc. Am. 63, 999-1006. https://doi.org/10.1093/cid/ciw451

Tcharkhtchi, A., Abbasnezhad, N., Zarbini Seydani, M., Zirak, N., Farzaneh, S., Shirinbayan, M. (2021). An overview of filtration efficiency through the masks: Mechanisms of the aerosols penetration. Bioact. Mater. 6, 106-122. https://doi.org/10.1016/j.bioactmat.2020.08.002

U.S. EPA (2011). Exposure Factors Handbook 2011 Edition (Final Report). U.S. Environmental Protection Agency, Washington, DC, EPA/600/R-09/052F, 2011.

U.S. Food and Drug Administration (FDA) (2004). Surgical masks-Premarket notification [510(k)] submissions. Guidance for Industry and FDA Staff. FDA-2003-D-0305. US Food and Drug Administration. https://www.fda.gov/regulatory-information/search-fda-guidance-document s/surgical-masks-premarket-notification-510k-submissions (accessed 20 April 2021).

Wang, C.S. (2001). Electrostatic forces in fibrous filters-A review. Powder Technol. 118, 166170. https://doi.org/10.1016/S0032-5910(01)00307-2

Yan, Y., Bayham, J., Richter, A., Fenichel, E.P. (2021). Risk compensation and face mask mandates during the COVID-19 pandemic. Sci. Rep. 11, 3174. https://doi.org/10.1038/s41598-02182574-w

Zangmeister, C.D., Radney, J.G., Vicenzi, E.P., Weaver, J.L. (2020). Filtration efficiencies of nanoscale aerosol by cloth mask materials used to slow the spread of SARS-CoV-2. ACS Nano 14, 9188-9200. https://doi.org/10.1021/acsnano.0c05025

Zhang, L., Peng, Z., Ou, J., Zeng, G., Fontaine, R.E., Liu, M., Cui, F., Hong, R., Zhou, H., Huai, Y., 
Chuang, S.K., Leung, Y.H., Feng, Y., Luo, Y., Shen, T., Zhu, B.P., Widdowson, M.A., Yu, H. (2013). Protection by face masks against influenza $A(H 1 N 1) p d m 09$ virus on trans-Pacific passenger aircraft, 2009. Emerg. Infect. Dis. 19, 1403-1410. https://doi.org/10.3201/eid1909.121765

Zhao, M., Liao, L., Xiao, W., Yu, X., Wang, H., Wang, Q., Lin, Y.L., Kilinc-Balci, F.S., Price, A., Chu, L., Chu, M.C., Chu, S., Cui, Y. (2020). Household materials selection for homemade cloth face coverings and their filtration efficiency enhancement with triboelectric charging. Nano Lett. 20, 5544-5552. https://doi.org/10.1021/acs.nanolett.0c02211 